\title{
Effects of the copper intrauterine device versus injectable progestin contraception on pregnancy rates and method discontinuation among women attending termination of pregnancy services in South Africa: a pragmatic randomized controlled trial
}

G. Justus Hofmeyr ${ }^{1}$, Mandisa Singata-Madliki ${ }^{1}$, Theresa A. Lawrie ${ }^{1,5^{*}}$, Eduardo Bergel ${ }^{4}$ and Marleen Temmerman ${ }^{2,3}$

\begin{abstract}
Background: The copper intrauterine device (IUD) is under-utilised in South Africa, where injectable progestin contraception (IPC) dominates contraception usage. There is a lack of robust comparative data on these contraceptive options to inform policy, programs, clinical counseling, and women's choices.

Methods: Within the context of a South African program to increase women's access to the IUD, we conducted a pragmatic, open-label, parallel-arm, randomised controlled trial of the IUD versus IPC at two South African hospitals. The target sample size was 7,000 women and the randomisation ratio was 1:1. The random sequence was computer-generated and group allocation was concealed in sealed, opaque, consecutively-numbered envelopes. Counselled, consenting women attending termination of pregnancy services were randomly assigned to IUD or IPC immediately post-termination. Condoms were promoted for the prevention of sexually-transmitted infections. The primary outcome was pregnancy; secondary outcomes were discontinuation, side-effects, and HIV acquisition and disease progression. Pregnancy and discontinuation outcomes are reported here.

Results: The trial closed early with 2,493 participants randomised (IUD =1,247, IPC =1,246), due to international concerns regarding a possible association between IPC and HIV acquisition. Median follow-up was 20 months; 982 and 1000 participants were followed up in the IUD and IPC groups, respectively. Baseline group characteristics were comparable. Pregnancy occurred significantly less frequently among women allocated to the IUD than IPC: 56/971 $(5.8 \%$ ) versus $83 / 992$ (8.4\%), respectively; risk ratio (RR) $0.69,95 \%$ confidence interval (CI) 0.50 to $0.96 ; P=0.025$. There were more protocol violations in the IUD group; however, discontinuation rates were similar between IUD and IPC groups (141/855 [16.5 \%] and 143/974 [14.7\%], respectively). Women in the IUD group were more likely to discontinue contraceptive use due to abdominal pain or backache and non-specific symptoms, and those in the IPC group due to oligo- or amenorhoea and lack of sexual activity.

(Continued on next page)
\end{abstract}

\footnotetext{
* Correspondence: tess@lawrie.com

'Effective Care Research Unit, Eastern Cape Department of Health/

Universities of the Witwatersrand, Walter Sisulu and Fort Hare, Mthatha,

Eastern Cape, South Africa

${ }^{5}$ Royal United Hospital, Bath, UK

Full list of author information is available at the end of the article
} 
(Continued from previous page)

Conclusions: The IUD was significantly more effective in preventing pregnancy than IPC. Efforts to expand contraception options and improve access to the IUD in settings where it is under-utilised are worthwhile. This trial shows that randomising long-acting, reversible contraceptives is feasible.

Trial registration: Pan African Clinical Trials Registry number PACTR201409000880157 (04-09-2014).

Keywords: DMPA, IUD, Random

\section{Background}

More than 4,000 terminations of pregnancy are performed each year in the East London/Mdantsanes area of South Africa and, in a survey of postnatal women attending local hospitals and clinics, two thirds of births were unintended [1]. Like most of South Africa, women utilising services at Frere and Cecelia Makiwane Hospitals and clinics have a limited choice of contraceptives and injectable progestin contraception (IPC), including depot medroxyprogesterone acetate (DMPA) and norethisterone enantate (NET), account for most contraception use. A common reason for discontinuation of contraception by women attending these services is dissatisfaction with the contraceptive method. The World Health Organization (WHO) continues to highlight the need for a wider variety of highly effective contraceptive methods to be available to women living in low and middle-income countries (LMICs) [2].

The copper intrauterine device (IUD) is a wellestablished, highly effective method of contraception; however, availability issues, staff capacity, provider attitude, and perception of side effects have held back its use in many LMICs, including South Africa [3-5]. In 2005, we initiated a program at Frere and Cecelia Makiwane Hospitals and clinics to expand contraceptive options to include the copper IUD (SMB ${ }^{\circ}$ Model $\mathrm{TCu}$ $380 \mathrm{~A}$ ), which was previously unavailable in our services. However, we found comparative data on the relative contraceptive effectiveness of the IUD and IPC to be insufficient for adequate counselling of prospective users: Limited evidence from a 2010 Cochrane review suggested that the IUD was more effective in preventing unintended pregnancy than hormonal contraception (including IPC), but had not been further elucidated [6]; and observational data were subject to confounding as contraceptive choice often varies according to factors related to the likelihood of pregnancy. To enhance the knowledge base for our counselling and promotion of wider contraception options, we designed a pragmatic trial within our routine contraceptive services to compare effectiveness, method discontinuation, and reasons for discontinuation, of the newly introduced method (IUD) with that of the most widely-used method (IPC). As secondary outcomes, we also assessed side effects,
HIV acquisition and HIV/AIDS disease progression, which we intend to report separately.

\section{Methods}

\section{Study design and participants}

The trial was a pragmatic, open-label, parallel arm randomised controlled trial conducted at two Eastern Cape hospitals, Frere Hospital (FH) and Cecelia Makiwane Hospital (CMH), in South Africa. Recruitment began on 6 July 2009 and ended on 8 November 2012. Women attending termination of pregnancy services at the study sites, who met the inclusion criteria and requested longterm contraception with no personal preference for a particular method, were counselled in their home language and offered participation in the trial. Women were eligible if they intended to continue contraception for at least one year, were $\geq 16$ years old, had no evidence of active pelvic infection on history and clinical examination, had no contraindications to IPC or IUD use, were prepared to use either method of contraception, understood the patient information form, and were willing to sign informed consent. All women were offered counselling and voluntary HIV testing at baseline and at followup, according to national health policy, using rapid tests or laboratory-based ELISA tests. Those with positive results were offered a CD4 count and treatment according to national health guidelines.

\section{Ethics, consent, and permissions}

Ethical approval for the trial was obtained from the University of the Witwatersrand Committee for Research on Human Subjects, South Africa, on the 25 April 2008, clearance certificate M080466. Signed informed consent to participate in the study was obtained from all participants.

\section{Randomisation}

An allocation list was prepared using a computergenerated random sequence in balanced blocks of variable size and with a randomisation ratio of 1:1. Allocation slips were inserted into consecutively-numbered, opaque envelopes and sealed by a member of staff not involved in the trial. The randomisation list was sealed in a signed envelope by the same staff member for safekeeping. The 
randomisation envelopes were distributed to the study sites in batches of 100 . Women who agreed to participate were entered onto a trial register and then enrolled by opening the next numbered, sealed envelope. The point of trial entry was at the opening of the envelope.

\section{Interventions}

Women were fully counselled about their allocated contraception method prior to its administration. In the IUD group, the IUD was inserted after termination of pregnancy, which was usually performed by manual vacuum aspiration by the hospital staff following routine procedures. Antibiotic prophylaxis was not used. The women were offered two options regarding the string placement after full counselling about the relative benefits of each: strings protruding from the cervix by about $2 \mathrm{~cm}$, or strings inserted completely within the uterine cavity or removed. The reason for offering these options is that many women in our services prefer their contraception to be confidential. In the IPC arm, women were counselled regarding the need for repeat injections at regular intervals. The first injection was administered by hospital staff according to routine practice and, in most instances, DMPA was used. However, as this was a pragmatic trial within the routine contraception services, allowance was made for the use of NET at the discretion of the provider. Women received no additional reminders to support continuation with the allocated contraception method, and no payments or other incentives were offered, other than payment of transport costs for the final visit at 12 months post-enrolment.

\section{Outcomes}

The primary outcome was pregnancy. Secondary outcomes were method discontinuation, side effects, HIV acquisition, and HIV/AIDS disease progression. Pregnancy and discontinuation outcomes are reported in this paper.

\section{Data collection}

Professional nurses who worked as research assistants completed a Good Clinical Practice certification course before trial commencement. Participant baseline details were entered onto a paper case report form (CRF), including demographic data, medical history, and baseline data relevant to the follow-up questionnaire (including sexual activity and potential side effects). Follow-up questionnaires were administered by the research assistants to participants by telephone at three, six, nine and 12 months after randomisation. The CRF and questionnaire were piloted before the main trial. The actual contraceptive method received after randomisation was collected retrospectively from family planning registers at the two hospitals. Participants were requested to attend an interview at the hospital 12 months after enrolment, failing which the interview was conducted by telephone. Initial and final contraceptive methods used were confirmed with the participant at the last interview. Other questions at this interview included whether the participant had been pregnant since joining the study, the outcome of the pregnancy, and what contraceptive methods had been used since joining the study, as well as reasons for discontinuation. Attempts to contact women who did not return for follow-up were continued until study closure in June 2014. Research assistants advised participants to attend the relevant health facility for any health concerns.

\section{Sample size and analysis}

The sample size was calculated based on the primary outcome. To detect a reduction in unplanned pregnancy from $2.5 \%$ to $1.5 \%$, we calculated that we needed 6,546 participants (alpha $=0.05$, beta $=0.20$; Epi Info ${ }^{\text {ti }}$ statistical software version 7). To allow for loss to follow-up, we planned to recruit a total sample size of 7,000 women.

Intention-to-treat analysis was performed for the primary outcome (pregnancy) using Epi Info ${ }^{\mathrm{Tm}}$ statistical software. Discontinuation rate was defined as the proportion of women who did not continue use of the allocated method to follow-up, out of the women initiating the allocated method and this analysis was, thus, per protocol.

Baseline characteristics were compared between enrolled groups, as well as those with follow up of 12 months or more. For categorical variables, the rates of outcome events were compared as risk ratios (RR) with $95 \%$ confidence intervals (CI). P-values were calculated using the Chi-squared test with Mantel-Haenszel correction or, for small numbers, the Fisher's exact test. $P$ values of less than 0.05 were regarded as statistically significant.

\section{Trial registration}

Registration of the protocol with the South African National Clinical Trials Register was undertaken on 15 September 2008 (Verification Code: 0-953). It was subsequently found not to have been logged by the system, and was re-registered with the Pan African Clinical Trials Registry on 4th September 2014 (PACTR201409000880157). (http://apps.who.int/trialsearch/ Trial2.aspx?TrialID=PACTR201409000880157).

\section{Results}

The trial closed early due to international concerns regarding a possible association between DMPA and HIV acquisition, following publication of an observational study reporting increased HIV acquisition among women who chose DMPA [7], and the subsequent plan by international stakeholders to conduct a large, multicentre trial that could address the DMPA/HIV question [8]. 
At closure, 2,493 of the target sample had accrued (36\%), with 1,247 and 1,246 women randomised to the IUD and IPC groups, respectively. An additional 19 randomisation envelopes could not be linked to participants (see CONSORT Fig. 1). Baseline data for the IUD and IPC groups were well matched (Table 1). Data on the primary outcome were available for 1,963 participants (78.7 \%). The median time to final interview was 20 months (interquartile range 15 to 28). In the IUD group, 56 out of 971 women (5.8\%) became pregnant during follow-up compared with 83 out of 992 women $(8.4 \%$ ) in the IPC group (risk ratio (RR) $0.69,95 \%$ confidence interval (CI) 0.50 to $0.96 ; P=0.025)$. There were no statistically significant differences between groups for the type of pregnancy outcome (Table 2).

Data on discontinuation were available for 982 women $(78.7 \%)$ in the IUD group and 1000 women $(80.3 \%)$ in the IPC group. In the IUD group, $855(87.1 \%)$ of the 982 women received an IUD as allocated; the other 127 women (12.9 \%) received a non-allocated method (protocol violations), which was an IPC in most (125) cases. In the IPC group, 974 out of 1000 women $(97.4 \%)$ received an IPC as allocated, with $85.5 \%$ receiving DMPA; the remainder received NET. Twenty-three out of the 26 women in the IPC group receiving a nonallocated method (protocol violations) received an IUD, and follow-up data did not distinguish between DMPA and NET. At the final follow-up interview, 729 women $(74.2 \%)$ out of 982 women in the IUD group were using an IUD, compared with 831 (83.1\%) out of 1000 women in the IPC group (intention-to-treat). Loss to follow-up was relatively high and, in the worse case scenario, if all those lost to follow-up were not using the allocated method, the percentage of women using the allocated

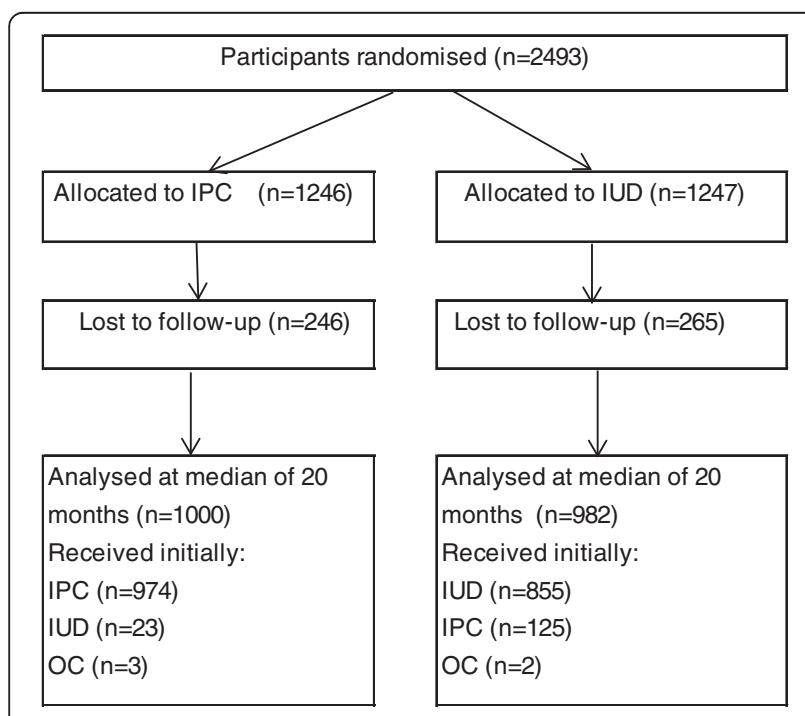

Fig. 1 CONSORT diagram showing the flow of participants method at follow-up in the IUD and IPC groups was $58.5 \%$ and $66.7 \%$, respectively. However, among women who initiated their allocated treatment as per protocol, discontinuation at a median follow-up of 20 months was not statistically significantly difference between the IUD and the IPC groups (141/855 women [16.5\%] versus 143/974 women [14.7 \%], respectively; RR 1.12, 95 \% CI 0.91 to $1.39 ; P=0.29$ ) (Table 3).

Women in the IUD group were more likely than those in the IPC group to discontinue contraceptive use due to abdominal pain or backache $(P=0.00005)$ and nonspecific symptoms $(P=0.004)$, and those in the IPC group were more likely to discontinue due to oligo-or amenorhoea $(P=0.004)$ and lack of sexual activity $(P=0.0002)$ (Table 4). Expulsion of the IUD was given as the reason for discontinuation in 26/135 (19.3\%) women who discontinued IUD use, giving an expulsion rate of $3 \%$. Two women in the IUD group and four women in the IPC group were reported to have died during the follow-up period. Information on these women is shown in Table 5.

\section{Discussion}

As mentioned above, during the conduct of our trial an observational study published findings of increased HIV acquisition among women who chose DMPA contraception, which gained wide media attention [7]. Trial investigators attended international stakeholder consultations, where there were calls for a large, multicentre trial to conclusively answer the questions on the effects of hormonal contraception on HIV acquisition and disease progression [2]. In such a trial, any evidence relating to DMPA should be robust and distinct from other longacting, progestin contraceptives. As the current pragmatic trial was unable to unequivocally answer the DMPA/HIV question due to the allowance of DMPA or NET in the IPC group, we stopped recruitment in November 2012. We followed up the reduced sample as planned with the aim of using these data to inform the design of the requisite, more robust trial [8].

Nevertheless, the sample size was adequate to assess the primary outcome due to the occurrence of higher than expected pregnancy rates in this trial. In contraceptive guidelines, contraceptive failure with typical use is considered to be about six pregnancies per 100 womenyears with IPC use, and one per 100 women-years with IUD use [9]. The estimate for IPC failure is supported by our findings, and those of a recent observational study of contraceptive use in South Africa, which reported pregnancy rates with IPC of 4.4 per 100 womenyears [3]. However, pregnancy rates in women allocated to IUD use in our study, although significantly lower with the IUD than IPC, were higher than expected. This was probably partly due to over-estimation on intentionto-treat analysis, as $13 \%$ of women allocated to the IUD 
Table 1 Baseline data expressed as numbers (\%) or mean values (standard deviation (SD))

\begin{tabular}{|c|c|c|c|c|}
\hline & \multicolumn{2}{|l|}{ IUD group } & \multicolumn{2}{|l|}{ IPC group } \\
\hline & $n(\%)$ or mean [SD] & N & $n(\%)$ or mean [SD] & N \\
\hline Age in years & $26.7[6.3]$ & 1234 & $26.4[6.1]$ & 1239 \\
\hline Weight in kgs & $71.7[17.9]$ & 1214 & $70.2[16.4]$ & 1220 \\
\hline Previous miscarriage & $107(8.7)$ & 1235 & $100(8.1)$ & 1232 \\
\hline Previous caesarean section & $163(13.3)$ & 1230 & $146(11.8)$ & 1236 \\
\hline Previous pelvic sepsis & 241 19.6) & 1230 & $237(19.2)$ & 1232 \\
\hline Hypertension & $14(1.1)$ & 1236 & $11(0.9)$ & 1242 \\
\hline Diabetes mellitus & $5(0.4)$ & 1236 & $6(0.5)$ & 1241 \\
\hline HIV positive & $236(21.2)$ & 1110 & $240(21.6)$ & 1118 \\
\hline \multicolumn{5}{|l|}{ Previous contraception use } \\
\hline$-I P C$ & $801(71.1)$ & 1126 & $824(72.5)$ & 1136 \\
\hline -oral contraceptive pill & $90(7.7)$ & 1176 & $64(5.4)$ & 1184 \\
\hline$-I U D$ & $2(0.2)$ & 1177 & $1(0.1)$ & 1188 \\
\hline
\end{tabular}

IUD intrauterine device, IPC injectable progestin contraception

group received IPC. Thus, our findings provide robust evidence that allocation to the IUD is more effective than allocation to IPC in preventing unintended pregnancies in South Africa. This has important implications for contraceptive policy in South Africa, where injectable progestin contraception accounts for the vast majority of contraception in the public sector and efforts to improve the method mix have had limited success.

A large study of more than 200,000 women from 14 developing countries reported a median probability of IUD discontinuation of $13.2 \%$ [10]. Higher rates of IUD and IPC discontinuation (and unintended pregnancy) have been observed among women with a history of termination of pregnancy [11-14]. In two post-termination cohorts of women who chose IPC, discontinuation rates as high as 64 per 100 woman-years, and $84 \%$ were reported $[13,14]$. As all the participants in our trial were recruited post-termination of pregnancy, this factor may have contributed to contraceptive failure and discontinuation in our trial.
Due to the pragmatic nature of this trial, loss to follow-up was relatively high, at around $20 \%$ in both intervention groups. This limitation could have lead to an under-estimation of method discontinuation due to differential loss of women who discontinued their allocated method. For this reason we reported the proportion of women using each method out of those with known follow up data, as well as for of the intervention groups as a whole. Baseline characteristics were comparable, therefore, loss to follow-up did not appear to have been differential between random allocation groups. Another limitation of the trial was the high number of protocol violations in the IUD group; $12.7 \%$ of women with follow-up data in the IUD group received the IPC after randomisation instead of the IUD. The reasons for protocol violations were not recorded, but could have been due to women declining the IUD after randomisation in favour of the more familiar method (IPC), or difficulties with IUD insertion, or provider barriers. Of the participants with data on previous contraceptive use,

Table 2 Pregnancy rates and outcomes

\begin{tabular}{|c|c|c|c|c|c|c|c|}
\hline & \multicolumn{2}{|c|}{ IUD group $(N=971)$} & \multicolumn{2}{|c|}{ IPC group $(N=992)$} & \multirow[t]{2}{*}{ RR } & \multirow[t]{2}{*}{$95 \% \mathrm{Cl}$} & \multirow[t]{2}{*}{$P$-value $(\mathrm{M}-\mathrm{H})$} \\
\hline & $n$ & $\%$ & $n$ & $\%$ & & & \\
\hline Pregnancy (total) & 56 & 5.8 & 83 & 8.4 & 0.69 & 0.50 to 0.96 & 0.025 \\
\hline \multicolumn{8}{|c|}{ Pregnancy outcome: } \\
\hline Birth & 29 & 3 & 41 & 4.1 & 0.72 & 0.45 to 1.15 & 0.17 \\
\hline Miscarriage & 3 & 0.3 & 3 & 0.3 & & & \\
\hline Termination & 2 & 0.2 & 2 & 0.2 & & & \\
\hline Ectopic & 0 & 0 & 1 & 0.1 & & & \\
\hline Ongoing & 16 & 1.6 & 29 & 2.9 & 0.56 & 0.31 to 1.03 & 0.059 \\
\hline Unknown & 6 & 0.6 & 7 & 0.7 & & & \\
\hline
\end{tabular}


Table 3 Allocated and actual contraception method and discontinuation

\begin{tabular}{|c|c|c|c|c|c|c|}
\hline \multirow[b]{3}{*}{ Actual method/s used } & \multicolumn{6}{|c|}{ Allocated method } \\
\hline & \multicolumn{3}{|l|}{ IUD } & \multicolumn{3}{|l|}{ IPC } \\
\hline & $N$ & $\%$ final method known & $\%$ total & $N$ & $\%$ final method known & $\%$ total \\
\hline Initial method used: IUD & 855 & $87.1 \%$ & $68.6 \%$ & 23 & $2.3 \%$ & $1.8 \%$ \\
\hline \multicolumn{7}{|l|}{ Initial method/final method: } \\
\hline IUD/IUD & 714 & $72.7 \%$ & $0.1 \%$ & 21 & $2.1 \%$ & $1.7 \%$ \\
\hline IUD/IPC & 32 & $3.3 \%$ & $2.6 \%$ & 0 & $0.0 \%$ & $0.0 \%$ \\
\hline IUD/OC & 4 & $0.4 \%$ & $0.3 \%$ & 0 & $0.0 \%$ & $0.0 \%$ \\
\hline IUD/Sterilisation & 1 & $0.1 \%$ & $0.1 \%$ & 0 & $0.0 \%$ & $0.0 \%$ \\
\hline IUD/none & 104 & $10.6 \%$ & $8.3 \%$ & 2 & & $0.2 \%$ \\
\hline Initial method used: IPC & 125 & $12.7 \%$ & $10.0 \%$ & 974 & $97.4 \%$ & $78.2 \%$ \\
\hline IPC/IPC & 105 & $10.7 \%$ & $8.4 \%$ & 831 & $83.1 \%$ & $66.7 \%$ \\
\hline IPC/IUD & 15 & $1.5 \%$ & $1.2 \%$ & 11 & $1.1 \%$ & $0.9 \%$ \\
\hline IPC/OC & 0 & $0.0 \%$ & $0.0 \%$ & 11 & $1.1 \%$ & $0.9 \%$ \\
\hline IPC/sterilisation & 0 & $0.0 \%$ & $0.0 \%$ & 3 & $0.3 \%$ & $0.2 \%$ \\
\hline IPC/none & 5 & $0.5 \%$ & $0.4 \%$ & 118 & $11.8 \%$ & $9.5 \%$ \\
\hline Initial method used: Other & 2 & $0.2 \%$ & $0.2 \%$ & 3 & $0.3 \%$ & $0.2 \%$ \\
\hline \multicolumn{7}{|l|}{ Initial method/final method: } \\
\hline OC/OC & 2 & $0.2 \%$ & $0.2 \%$ & 3 & $0.3 \%$ & $0.2 \%$ \\
\hline Final method known & 982 & & $78.7 \%$ & 1000 & & $80.3 \%$ \\
\hline Final method unknown & 265 & & $21.3 \%$ & 246 & & $19.7 \%$ \\
\hline Total randomised & 1247 & & & 1246 & & \\
\hline
\end{tabular}

IUD intrauterine device, IPC injectable progestin contraception

Table 4 Discontinuation and reasons

\begin{tabular}{|c|c|c|c|c|c|c|c|}
\hline Reason for discontinuation (total) & IUD group & & IPC group & & RR & $95 \% \mathrm{Cl}$ & $P$-value $(\mathrm{M}-\mathrm{H})$ \\
\hline & & 135 & & 147 & & & \\
\hline Want baby/married & 19 & 14.1 & 26 & 17.7 & 0.8 & 0.46 to 1.37 & 0.41 \\
\hline Fell pregnant & 3 & 2.2 & 1 & 0.7 & & & \\
\hline Abdominal pain/backache & 21 & 15.6 & 3 & 2 & 7.62 & 2.33 to 25 & 0.00005 \\
\hline Heavy menstruation & 9 & 6.7 & 13 & 8.8 & 0.75 & 0.33 to 1.71 & 0.5 \\
\hline Scanty/no menstruation & 0 & 0 & 9 & 6.1 & 0 & NE & 0.004 (FE) \\
\hline Not sexually active & 11 & 8.1 & 36 & 24.5 & 0.33 & 0.18 to 0.63 & 0.0002 \\
\hline Weight gain & 0 & 0 & 3 & 2 & & & \\
\hline Weight loss & 1 & 0.7 & 0 & 0 & & & \\
\hline Partner request & 1 & 0.7 & 0 & 0 & & & \\
\hline Discomfort during intercourse & 1 & 0.7 & 0 & 0 & & & \\
\hline Infection & 2 & 1.5 & 0 & 0 & & & \\
\hline Itchy vulvae/discharge & 2 & 1.5 & 0 & 0 & & & \\
\hline Headaches & 0 & 0 & 1 & 0.7 & & & \\
\hline Non-specific symptoms & 12 & 8.9 & 2 & 1.4 & 6.5 & 1.49 to 29 & 0.004 \\
\hline Using condoms & 1 & 0.7 & 4 & 2.7 & & & \\
\hline Suspected cancer & 1 & 0.7 & 0 & 0 & & & \\
\hline No reason given & 25 & 18.5 & 49 & 33.3 & 0.53 & 0.35 to 0.82 & 0.003 \\
\hline IUD came out & 26 & 19.3 & 0 & 0 & & & \\
\hline
\end{tabular}


Table 5 Women reported to have died

\begin{tabular}{llllllll}
\hline Age $(\mathrm{y})$ & Baseline HIV status & Weight (Kg) & Group allocation & Option received & Date enrolled & Approximate date of death & Reported cause of death \\
\hline 31 & Unknown & 81 & IUD & IUD & 02-Sep-09 & 2012 & Motor vehicle accident \\
24 & Unknown & 65 & IUD & IUD & 24-Feb-10 & 15-May-10 & Motor vehicle accident \\
18 & Negative & 67 & IP & DMPA & 13-Jul-10 & Dec-11 & Eclampsia \\
32 & Positive & $?$ & IP & DMPA & 08-Nov-10 & 18-Dec-11 & Knife wound/s \\
26 & Positive & 49 & IP & DMPA & 10-Nov-11 & 10-Feb-12 & HIV-related infection \\
20 & Negative & 60 & IP & NET & 07-Aug-12 & May-13 & Asthmatic attack \\
\hline
\end{tabular}

1625 had previously used IPC versus only three previously using the IUD (Table 3 ). Whilst protocol violations may have underestimated differences between contraceptive methods on pregnancy rates, in intention-totreat analysis they are unlikely to have over-estimated effect differences.

While individual choice is a cornerstone of contraceptive services, this trial demonstrates that after counseling on different contraceptive options many women did not have a specific preference for a contraceptive method and were willing to agree to randomisation without any financial remuneration or other benefits. Although initial compliance in the IUD group was sub-optimal (87\%), the acceptability of randomisation is supported by the fact that discontinuation of allocated methods were comparable with observational studies in which women had chosen their contraceptive method [10-14]. No trialrelated steps were taken to support adherence to the allocated contraception method, and no payments or other incentives were offered, other than payment of transport costs for the final visit at 12 or more months postrandomisation. In addition, the provision and follow up of the allocated contraceptive method took place within the routine health service. The advantage of this was that relative discontinuation rates and contraceptive effectiveness were more likely to reflect the 'typical-use' effect of these methods in routine practice (though absolute rates might differ from women choosing a specific method). The disadvantage was that follow-up was less efficient and protocol violations were higher than might have been achieved with a more intensive research intervention.

\section{Conclusions}

The IUD was more effective than IPC in a posttermination of pregnancy setting in South Africa. Disontinuation rates with the IUD were comparable to the more familiar and widely accepted IPC, suggesting that efforts to expand contraception method choice and overcome user and provider barriers to IUDs are worthwhile. Study limitations such as protocol violations may have led to an under-estimation of differences in the primary outcome, but over-estimation is unlikely. This research supports the feasibility of conducting a large, multi-centre randomised trial to address the DMPA/HIV question.
Competing interests

GJH, MS and MT are members of the Expanding Contraceptive Health Options $(\mathrm{ECHO})$ consortium who are planning a randomised trial of contraceptive methods and HIV acquisition.

\section{Authors' contributions}

GJH contributed to study design, data analysis, and the drafting and revision of the report. MS contributed to study design, project management, data collection. TAL contributed to data analysis, interpretation, and drafting and revision of the report. MT contributed to the drafting and revision of the report. EB contributed to study design. All authors reviewed and approved the final manuscript.

\section{Acknowledgements}

The costs of this study were internally funded by the Effective Care Research Unit (ECRU), East London, South Africa. We gratefully acknowledge the women who supported the trial by way of participation, and staff at $\mathrm{FH}$ and $\mathrm{CMH}$ who contributed to the contraceptive care of women in the study. We acknowledge the contributions of Mrs Ana Nondlwana and other research staff of the Effective Care Research Unit.

\section{Funding}

Contraceptive care was provided by the routine clinical services and research costs were covered by the Effective Care Research Unit, East London, South Africa.

\section{Author details}

${ }^{1}$ Effective Care Research Unit, Eastern Cape Department of Health/ Universities of the Witwatersrand, Walter Sisulu and Fort Hare, Mthatha, Eastern Cape, South Africa. ${ }^{2}$ Reproductive Health and Research, World Health Organization, Geneva, Switzerland. ${ }^{3}$ International Centre for Reproductive Health, Ghent University, Ghent, Belgium. ${ }^{4}$ Institute for Clinical Effectiveness and Health Policy (IECS), Buenos Aires, Argentina. ${ }^{5}$ Royal United Hospital, Bath, UK.

Received: 12 February 2016 Accepted: 23 March 2016

Published online: 18 April 2016

References

1. Mshweshwe TN, Mgoli A, Hofmeyr GJ, Mangesi L. Assessing the prevalence of unwanted pregnancies and barriers to use of preventive measures in East London. 27th Conference on Priorities in Perinatal Care in Southern Africa. Gauteng: Indaba Hotel; 2008. p. 11-4.

2. World Health Organization. Programmatic and research considerations for hormonal contraception for women at risk of HIV and women living with HIV. 2012. WHO/RHR/12.09.

3. Moodley J, Naidoo S, Wand H, Ramjee G. Contraception use and impact on pregnancy prevention in women participating in an HIV prevention trial in South Africa. J Fam Plan Repro Health Care. 2016;42(1):5-11.

4. Chakraborty NM, Murphy C, Paudel M, Sharma S. Knowledge and perceptions of the intrauterine device among family planning providers in Nepal: a crosssectional analysis by cadre and sector. BMC Health Serv Res. 2015:15:39.

5. Agha S, Fareed A, Keating J. Clinical training alone is not sufficient for reducing barriers to IUD provision among private providers in Pakistan. Repro Health. 2011;8:40.

6. Hofmeyr GJ, Singata M, Lawrie TA. Copper containing intra-uterine devices versus depot progestogens for contraception. Cochrane Database of 
Systematic Reviews 2010, Issue 6. Art. No.: CD007043. doi:10.1002/14651858. CD007043.pub2.

7. Heffron R, Donnell D, Rees H, Celum C, Mugo N, Were E, et al. Use of hormonal contraceptives and risk of HIV-1 transmission: a prospective cohort study. Lancet Infect Dis. 2012;12(1):19-26.

8. Cates W, The Evidence for Contraceptive Options and HIC Outcomes (ECHO) Consortium. Research on Hormonal Contraception and HIV. Lancet. 2014;383:303-4.

9. US Department of Health and Human Services. Centers for Disease Control and Prevention. Effectiveness of Family Planning Methods. http://www.cdc. gov/reproductivehealth/UnintendedPregnancy/PDF/Contraceptive_ methods_508.pdf (accessed 27 January 2016).

10. Ali MM, Park MH, Ngo TD. Levels and determinants of switching following intrauterine device discontinuation in 14 developing countries. Contraception. 2014;90(1):47-53.

11. Cohen R, Sheeder J, Arango N, Teal SB, Tocce K. Twelve-month contraceptive continuation and repeat pregnancy among young mothers choosing post-delivery contraceptive implants or postplacental intrauterine devices. Contraception. 2015. doi:10.1016/j.contraception.2015.10.001.

12. Modey EJ, Aryeetey R, Adanu R. Contraceptive discontinuation and switching among Ghanaian women: evidence from the Ghana Demographic and Health Survey, 2008. Afr J Reprod Health. 2014;18(1):84-92.

13. Sonalkar S, McClusky J, Hou MY, Borgatta L. Administration of depot medroxyprogesterone acetate on the day of mifepristone for medical abortion: a pilot study. Contraception. 2015;91(2):174-7.

14. Puri M, Henderson JT, Harper $\mathrm{CC}$, et al. Contraceptive discontinuation and pregnancy postabortion in Nepal: a longitudinal cohort study. Contraception. 2015:91(4):301-7.

\section{Submit your next manuscript to BioMed Central and we will help you at every step:}

- We accept pre-submission inquiries

- Our selector tool helps you to find the most relevant journal

- We provide round the clock customer support

- Convenient online submission

- Thorough peer review

- Inclusion in PubMed and all major indexing services

- Maximum visibility for your research

Submit your manuscript at www.biomedcentral.com/submit

) Biomed Central 